\title{
The simulation of the heat transfer on a plate with a precrack
}

\author{
Zhenyu Zhang ${ }^{1}$, Qiwen Liu ${ }^{1}$, Lisheng Liư ${ }^{2}$, Fang Wang ${ }^{1}$ \\ ${ }^{1}$ Department of Engineering Structure and Mechanics, Wuhan University of Technology, Wuhan, \\ 430070, China \\ ${ }^{2}$ State Key Laboratory of Materials Synthesis and Mechanics, Wuhan University of Technology, \\ Wuhan, 430070, China
}

Keywords: Peridynamic theory, crack, heat transfer, nonlocal methods.

\begin{abstract}
Peridynamic is a nonlocal theory based on the integral equations rather than the partial differential equation in classic theory, hence it is more suit for modelling the discontinuities areas, such as cracks, damage and so on. In this paper, we did a simulation of the heat transfer on a plate with a precrack based on the Peridynamic theory. The temperature and heat flux distribution is analyzed.
\end{abstract}

\section{Introduction}

It is very hard to deal with the discontinuous of physical field, since the continuum mechanics relies on the partial differential equations. The reason of this problem is that, the continuum mechanics assumes that only neighboring material points can interact with each other, so the equilibrium equation of a point in classic theory are some partial differential equations. However, this equations does not exist on those discontinue areas.

The Peridynamic theory is a very good way to deal with this troubles. It is a nonlocal theory proposed by Silling in 2000[1], in which two non-neighboring points can interact with each other directly. This long-range interaction make the motion equation in the form of integral, which is very suit to model those discontinue problems.

Since this method is proposed, it is used widely to model different kind of discontinues, such as the membrane deformation and failure with a precrack[2], the impact of a sphere on a brittle target[1], the Charpy V-notch test [3], the damage in plain concrete [4], and many other aspects. With all these practical application, the Peridynamic has shown its adaptability in many domain. The Peridynamic are also used in other physical field, such as heat conduction [5, 6], thermal-solid interaction, thermoelectric coupling and so on.

In this paper, we did a simulation of heat transfer on a plate with a precrack with the method proposed by Bobaru [6]. And analyzed the temperature and heat flux.

\section{The review of Peridynamic}

The Peridynamic formulation for solid mechanic. In Peridynamic theory, two non-neighboring points can interact with each other directly, this interaction is called a bond. The longest distance for two points that can interact with each other is called the horizon. The long-range interaction is named as the bond force $\mathbf{f}$, which relies on the relative position $\xi$ of two point in reference configuration and the relative displacement $\boldsymbol{\eta}$ at time

$$
\mathbf{f}=\mathbf{f}(\xi, \boldsymbol{\eta})
$$

Where,

$$
\begin{gathered}
\xi=\mathbf{x}^{\prime}-\mathbf{x} \\
\boldsymbol{\eta}=\mathbf{u}\left(\mathbf{x}^{\prime}, t\right)-\mathbf{u}(\mathbf{x}, t)
\end{gathered}
$$

Where, $\mathbf{x}$ is a given point and $\mathbf{x}^{\prime}$ is a point that has a long-range interaction with $\mathbf{x}, \mathbf{u}$ is the displacement vector field. So the acceleration of any point located is

$$
\rho \ddot{\mathbf{u}}(\mathbf{x}, t)=\int_{\mathbf{H}_{\mathbf{x}}} \mathbf{f}\left(\mathbf{u}\left(\mathbf{x}^{\prime}, t\right)-\mathbf{u}(\mathbf{u}, t), \mathbf{x}^{\prime}-\mathbf{x}\right) d V_{\mathbf{x}^{\prime}}+\mathbf{b}(\mathbf{x}, t)
$$


Where $\mathbf{b}$ is the prescribed body force density. And $\mathbf{H}_{\mathbf{x}}$, named as a neighborhood, is a sub-region in the reference, and is defined as

$$
\mathbf{H}_{x}=\left\{x^{\prime} \in \Omega:\left|\mathbf{x}-\mathbf{x}^{\prime}\right|<\delta\right\},
$$

The Peridynamic formulation for heat conduction. Similar to the formulation for solid mechanic, a thermal conductor bond was build first in heat conduction. As shown in Fig 1, an infinite plane with the thickness of $d$, and two parallel surfaces $p$ and $p$ was considered. These two surfaces are kept at two different temperatures, $\theta$ and $\hat{\theta}$. And a cylinder is considered, the cross sectional area are is $s$. With the assumption that there is no heat flows through the side face $A$, the thermal balance equation is

$$
\rho c d \frac{\partial \theta_{a}}{\partial t}=\frac{\kappa(\hat{\theta}-\theta)}{d}
$$

Where,

$\theta_{a}$ : The average temperature in the cylinder;

$\kappa$ : The material's thermal conductivity;

$\rho$ : Mass density;

$c$ : Specific heat capacity.

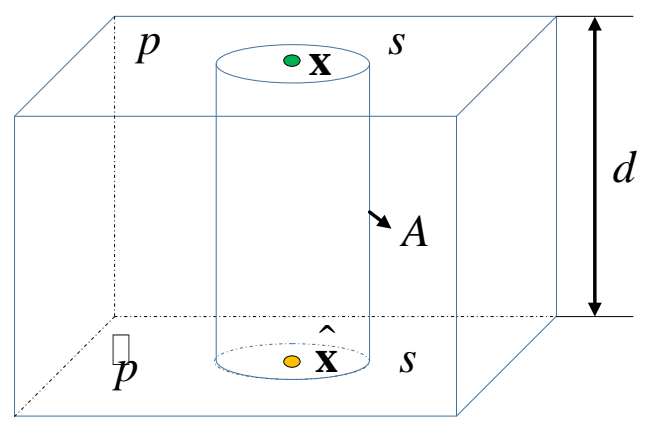

Fig 1: The deformation of a Peridynamic bond

For a body occupying a region $\Omega$ in three dimensional space, and composed of material points that have relevant mass and volume. Each material points is connected to the rest of the points in the body $\Omega$ via a t-bond, short for thermal conductor bond. It is reasonable to assume that the interaction distance for each material is limited to a certain number $\delta$, which made the interaction region of a point to be a spherical in three dimensional space.

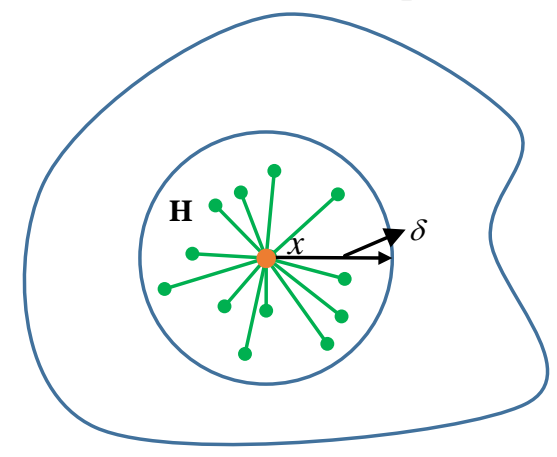

Fig 2: The deformation of a Peridynamic bond

The thermal balance equation for a point in three dimensional space with a neighborhood of $\mathbf{H}$ can be got by an integration over its neighborhood. 


$$
\int_{\mathbf{H}} \rho c \frac{\partial \theta_{a}(\mathbf{x}, \hat{\mathbf{x}}, t)}{\partial t} d V_{\hat{\mathbf{x}}}=\int_{\mathbf{H}} K(\mathbf{x}, \hat{\mathbf{x}}) \frac{\theta(\hat{\mathbf{x}}, t)-\theta(\mathbf{x}, t)}{|\hat{\mathbf{x}}-\mathbf{x}|^{2}} d V_{\hat{\mathbf{x}}}
$$

With the assumption that temperature at point $\mathbf{x}$ is the average temperature in all t-bond connected at $\mathbf{x}$, the thermal balance equation becomes

$$
\rho c \frac{\partial \theta(\hat{\mathbf{x}}, t)}{\partial t}=\int_{\mathbf{H}} k(\mathbf{x}, \hat{\mathbf{x}}) \frac{\theta(\hat{\mathbf{x}}, t)-\theta(\mathbf{x}, t)}{|\hat{\mathbf{x}}-\mathbf{x}|^{2}} d \hat{\mathbf{x}}+w(\mathbf{x}, t)
$$

Where $k(\mathbf{x}, \hat{\mathbf{x}})=K(\mathbf{x}, \hat{\mathbf{x}}) / V_{\mathbf{H}}$ defined to be the micro-conductivity of the t-bond $(\mathbf{x}, \hat{\mathbf{x}})$, and $w(\mathbf{x}, t)$ is the rate of the heat generation per unit volume per unit time function.

\section{The numerical method}

In this paper, the region is discretized in to nodes, each with a known volume in the reference configuration with a mesh free method. This mesh free method is used since there is no elements or other geometrical connections between these nodes, so this method is more suit for the large deformation and complex geometry. So the thermal balance equation replaces the integral by a finite sum:

$$
\rho c \dot{\theta}\left(x_{i}, t\right)=\sum_{j=1}^{n} k\left(x_{i}, x_{i j}\right) \frac{\theta\left(x_{i j}, t\right)-\theta\left(x_{i}, t\right)}{\left(x_{i j}-x_{i}\right)^{2}} V_{i j}
$$

A forward Euler integration method is used for the temperature at time $t+\Delta t$ :

$$
\theta(x, t+\Delta t)=\theta(x, t)+\dot{\theta}(x, t) \Delta t
$$

Where a constant time step size is assumed.

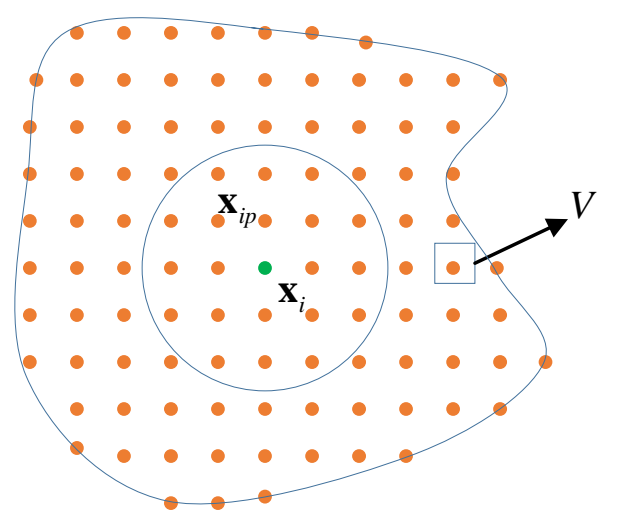

Fig 3: The deformation of a Peridynamic bond

\section{The numerical method}

In this section, the ability of the method to deal with a heat transfer for those discontinue materials is demonstrated by a representative model. In this model, a square plate with the side length $l=50 \mathrm{~mm}$ contains a center crack, which length is $a=20 \mathrm{~mm}$. The material property are shown in table 1 . A couple of non-adjacent sides are hold at different temperature $\theta_{1}$ and $\theta_{2}$. The other two sides are set to be convective boundary, the temperature out of the plate is set to be a constant of $\theta_{3}=50^{\circ} \mathrm{C}$. The plate is take discretized into nodes with a grid constant grid spacing $\Delta x=0.10 \mathrm{~mm}$, and a constant horizon size $\delta=0.31 \mathrm{~mm}$ is assumed which is slightly greater than $3 \Delta x$. 
Table 1: The Material properties

\begin{tabular}{cr}
\hline material property & value \\
\hline Density & $5000 \mathrm{~kg} / \mathrm{m}^{3}$ \\
coefficient of heat conduction & $350 \mathrm{~W} / \mathrm{m} \cdot \mathrm{K}$ \\
convective heat transfer & $5 \mathrm{~W} / \mathrm{m}^{2} \cdot \mathrm{K}$ \\
coefficient & \\
Heat capacity & $385 \mathrm{~J} / \mathrm{kg} \cdot \mathrm{K})$ \\
\hline
\end{tabular}

The flow plot shows the solution of temperature and the heat flow in Peridynamic theory, after the temperature field tend to be stable. Totally, the temperature is linear distributed on the field, except on the area near the crack. With the effect of impeding heat transfer, the temperature distribution is quite nonuniform near the crack. The heat flux near the crack tip is obviously large than other area on the crack, and is very small on the middle point of the crack since the impeding of the crack and the symmetric distribution of geometry and the boundary condition.

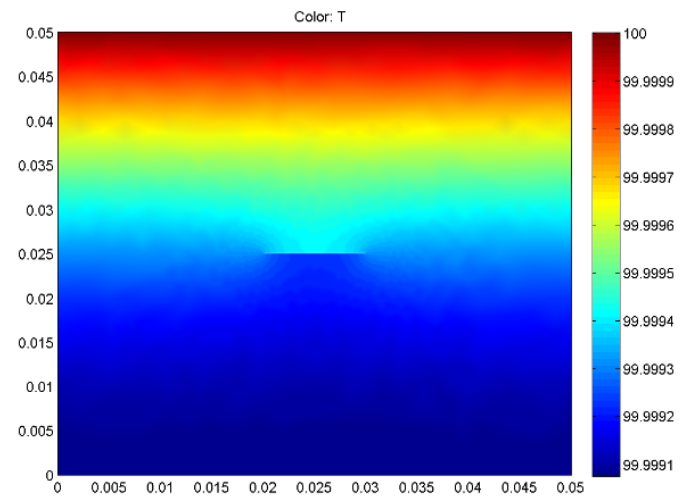

Fig 4: The deformation of a Peridynamic bond

\section{Conclusions}

In this paper, a thermal conductor bond is used for modeling the heat transfer in a plate with a precrack. An example of heat transfer is advanced. Temperature and heat flux are analyzed. Generally the temperature distribution is linear on the plate except area near the crack. The heat flux is obviously large than other area on the crack. The method is suitable for modeling the discontinue problems.

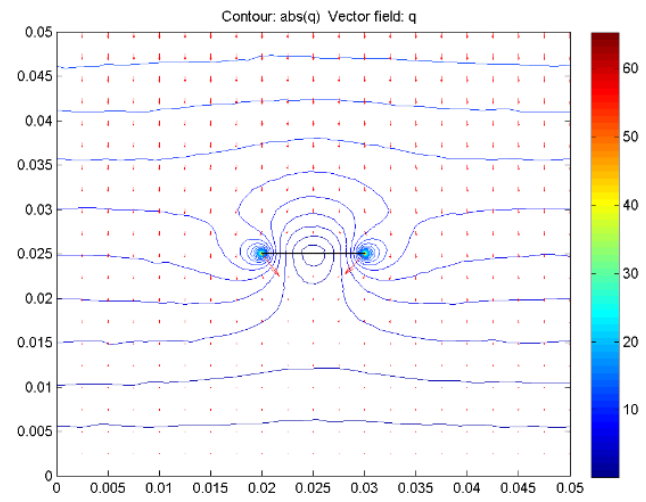

Fig 5: The deformation of a Peridynamic bond 


\section{Acknowledgements}

This work is financially supported by the Independent Innovation Research Fund Project of Wuhan University of Technology (No. 2014-zy-168). The corresponding author is Qiwen Liu.

\section{References}

[1] S. A. Silling, E. Askari, A Meshfree Method Based on the Peridynamic Model of Solid Mechanics, Computers and Structures 83(2005) 1526-135.

[2] S. A. Silling, F. Bobaru, Peridynamic Modeling of Membranes and Fibers, International Journal of Non-linear Mechanics 40(2005) 395-409.

[3] S. A. Silling, E. Askari, Peridynamic Modeling of Impact Damage,PVP-Vol.489, Problems Involving Thermal-Hydraulics, Liquid Sloshing, and Extreme Loads on Structures, July 2529,2004,San Diego, Caledonia, USA.

[4] Water, Gerstle, Nicolas Sau, Stewart Silling, Peridynamic Modeling of Plain and Reinforced Concrete Structures, $18^{\text {th }}$ International conference on structural mechanics in reactor technology (SMIRT 18), Beijing, Chine, August 7-12,2005.

[5] Florin Bobaru, Monchai Duangpanya, A Peridynamic Formulation for Transient Heat Conduction in Bodies with Evolving Discontinuities, Journal of Computational Physics, 231(2012) 2764-2785.

[6] Florin Bobaru, Monchai Duangpanya, the Peridynamic Formulation for Transient Heat Conduction. International Journal of Heat and Mass Transfer, 53(2010) 4070-4059. 